\title{
Ephedra-Treated Donor-Derived Gut Microbiota Transplantation Ameliorates High Fat Diet-Induced Obesity in Rats
}

\author{
Jing-Hua Wang ${ }^{1,2}$, Bong-Soo Kim ${ }^{3}$, Kyungsun Han ${ }^{1}$ and Hojun Kim ${ }^{1, *}$ \\ 1 Department of Rehabilitation Medicine of Korean Medicine, Dongguk University, 814 Siksa, Goyang, \\ Gyeonggi-do 10326, Korea; ewccwang@gmail.com (J.-H.W.); latortue@hanmail.net (K.H.) \\ 2 Key Laboratory of Xin'an Medicine, Ministry of Education, Anhui University of Traditional Chinese \\ Medicine, Meishan Road 103, Hefei 230038, China \\ 3 Department of Life Sciences, Hallym University, Chuncheon, Gangwon-do 24252, Korea; \\ bongsoo.km@gmail.com \\ * Correspondence: kimklar@gmail.com or kimklar@dongguk.ac.kr; Tel.: +82-31-961-9111
}

Academic Editor: Paul B. Tchounwou

Received: 17 March 2017; Accepted: 15 May 2017; Published: 23 May 2017

\begin{abstract}
Changes in gut microbiota (GM) are closely associated with metabolic syndrome, obesity, type 2 diabetes and so on. Several medicinal herbs, including Ephedra sinica (Es), have anti-obesity effects that ameliorate metabolic disorders. Therefore, in this study we evaluated whether Es maintains its anti-obesity effect through Es-altered gut microbiota (EsM) transplantation. GM was isolated from cecal contents of Es treated and untreated rats following repeated transplants into obese rats via oral gavage over three weeks. High-fat-diet (HFD)-induced obese rats transplanted with EsM lost significant body weight, epididymal fat, and perirenal fat weight, but no remarkable changes were observed in abdominal fat, liver, cecum weight and food efficiency ratio. In addition, treatment with EsM also significantly lowered the fasting blood glucose, serum insulin level, and insulin resistance index. Meanwhile, EsM transplantation significantly reduced gene expression of proinflammatory cytokines interleukin-1 and monocyte chemotactic protein-1. Rats treated with EsM also showed changed GM composition, especially blautia, roseburia and clostridium, significantly reduced the level of endotoxin and markedly increased the acetic acid in feces. Overall, our results demonstrated that EsM ameliorates HFD-induced obesity and related metabolic disorders, like hyperglycemia and insulin resistance, and is strongly associated with modulating the distribution of GM, enterogenous endotoxin and enteral acetic acid.
\end{abstract}

Keywords: ephedra; microbiota; transplantation; high fat diet; obesity

\section{Introduction}

Obesity was officially recognized as a disease by the American Medical Association in 2013 [1]. According to epidemiological findings from the World Health Organization, obesity, as a typical metabolic disorder, is becoming an intractable issue worldwide [2]. In general, obesity is caused by imbalance of energy intake and expenditure [3]. Therefore, reduction of energy intake and/or increase of basal metabolic rate (BMR) has been simplistically regarded as a therapeutic solution for controlling body weight gain [4]. However, both compulsive calorie restriction and permanent elevation of the BMR are extremely difficult in practice. Even invasive bariatric surgery and pharmacotherapy produce a variety of side effects and complications in Western medicine. Consequently, the need for an effective therapy for weight-loss with fewer or no adverse side effects is urgent.

Further, adipose tissue not only stores fat as a form of lipid but exerts sophisticated endocrine, autocrine, and paracrine functions to maintain energy homeostasis [5]. Large amounts of adipokines 
secreted by excessive adipocytes, such as interleukin (IL)-10, monocyte chemotactic protein-1 (MCP-1) [6,7], C-reactive protein [8], continuously promote low-grade inflammation resulting in a cascade of metabolic disorders [9]. Thus, obesity is characterized as a chronic and systemic inflammatory disease [10]. In addition, increasing scientific evidence demonstrates that a high-fat-diet (HFD) causes structural alteration of gut microbiota resulting in increased gastrointestinal permeability and endotoxemia [11]. Furthermore, metabolic endotoxemia evidently initiates obesity and insulin resistance [12,13]. That is, gut microbiota play a crucial role in metabolism as well as adipogenesis [14]. Interestingly, after fecal microbiota transplantation from a healthy but overweight donor, body weight gain was observed in a human subject [15]. Recent findings further revealed that gut microbiota impact host metabolism by differential DNA methylation as an epigenetic regulator [16]. Hence, effective modulation of gut microbiota might be a promising strategy for management of metabolic disorders, including obesity.

As a kind of therapeutic intervention, many medicinal herbs, formulas, and herbal derived components, including Flos lonicera [17], Rhizoma coptidis [18], Gegen Qinlian Decoction (composed of Radix puerariae, Radix scutellariae, Rhizoma coptidis, and honey-fried licorice root) [19], and berberine [20], were verified to exert beneficial efficacy on alleviating obesity and other chronic metabolic disorders via modulation of gut microbiota. Ephedra sinica (Es), also named ma huang, is commonly used in Asia as a traditional medicine for the treatment of common cold, flu, asthma, arthralgia, and edema for thousands of years [21]. Currently, Es and its principal active constituent, ephedrine, are commonly used as food additives for reducing body weight worldwide due to its powerful sympathomimetic action [22]. Several studies reported on the effects of Es on body weight loss in both obese human subjects and HFD-induced obese animal models associated with alteration of gut microbiota and regulation of adipokines [23,24]. Nevertheless, it is still undetermined whether Es itself or Es altered gut microbiota (EsM) effectively induce loss of body weight.

Therefore, in the current study we tried to verify the anti-obesity effect of Es through regulation of gut microbiota and to determine how gut microbiota can be modified by Es. We estimated the anti-obesity effect of intestinal microbiota regulated by Es and its possible mechanism.

\section{Materials and Methods}

\subsection{Aqueous Extraction of Ephedra sinica}

Korean pharmacopoeia standard Es was obtained from Dongguk University Ilsan International Hospital (Goyang, Korea). After washing with distilled water (DW) and oven-dying for $12 \mathrm{~h}, 500 \mathrm{~g}$ of Es was boiled in $4 \mathrm{~L}$ of DW for $4 \mathrm{~h}$ and filtered using a 300-mesh filter $(50 \mu \mathrm{m})$. The water extract was concentrated using a vacuum rotary evaporator following lyophilization at $-70{ }^{\circ} \mathrm{C}$ and stored at $-20^{\circ} \mathrm{C}$ for future use.

\subsection{High-Performance Liquid Chromatography (HPLC)}

HPLC-based fingerprinting was accomplished using two-dimensional HPLC (Agilent HP1200, Agilent Technologies, San Jose, CA, USA). Ephedrine (Sigma-Aldrich, St. Louis, MO, USA), as a principal component of Es, was used as a standard. Briefly, $100 \mu \mathrm{g}$ of water extract of Es, $10 \mu \mathrm{g}$ of ephedrine and $200 \mathrm{mg}$ of rat fecal samples from the end of microbiota preparation experiment were dissolved in $1 \mathrm{~mL} \mathrm{50 \%} \mathrm{of} \mathrm{methanol} \mathrm{separately.} \mathrm{After} \mathrm{sonication} \mathrm{and} \mathrm{centrifuging,} \mathrm{the} \mathrm{supernatant} \mathrm{of}$ samples were filtrated by $0.45 \mu \mathrm{m}$ membrane filter (Sartorius AG, Goettingen, Germany) and prepared for HPLC analysis. The Agilent HPLC system consisted of a quaternary pump, on-line solvent degasser, Agilent sample manager/column heater module, and Agilent UV detector. An Agilent Eclipse XDB-C18 $(5 \mathrm{~mm}, 4.6 \mathrm{~mm} \times 250 \mathrm{~mm})$ column was used and the compounds were eluted with solvents composed of acetonitrile: water: phosphoric acid (40:60:0.1 $v / v / v)$ containing sodium lauryl sulfate at a concentration of $5 \mathrm{~g} / \mathrm{L}$ as an ion-pairing agent. Peak identity of ephedrine in the samples was confirmed by comparison of spectrum and retention time. HP1200 ChemStation software (Agilent 
Technologies, Santa Clara, CA, USA) was applied and all chromatograms were obtained using a wavelength of $215 \mathrm{~nm}$.

\subsection{Animals and Experimental Design}

The animal experiments were performed according to the international guidelines (Guide for the Care and Use of Laboratory Animals, Institute of Laboratory Animal Resources, Commission on Life Sciences, National Research Council, USA; National Academy Press: Washington DC, 1996). The animal experiment protocols were reviewed and approved by the Institutional Animal Ethical Committee of Dongguk University (approval number: 2013-0580). All surgical procedures were performed under Zoletil (tiletamine-zolazepam, Virbac, Carros, France) and Rompun (xylazine-hydrochloride, Bayer, Leverkusen, Germany) combination $(1: 1, v / v)$ anesthesia. The detailed experimental schedule is as follows.

The first step was the gut microbiota preparation study (MP) (Figure 1A): 24 male 6-week old Sprague-Dawley (SD) rats were purchased from Orient Bio (Seongnam, Korea). HFD and normal diet (AIN-93G, Table 1) were obtained from Feedlab (Guri, Korea). The animals were acclimatized for one week at a controlled temperature $\left(20 \pm 2{ }^{\circ} \mathrm{C}\right)$, relative humidity $(40-60 \%)$ and a $12 \mathrm{~h}$ light-dark cycle with a commercial normal chow diet (Soyagreentec, Hwaseong, Korea) before the experiment. Rats were assigned randomly to three groups of eight each, as follows: MP normal, MP HFD, and MP Es group. Rats in the MP normal group were given the normal diet (AIN-93G, Table 1) ad libitum continuously for six weeks. Rats in the MP HFD and MP Es group were given commercial HFD with the same schedule as the MP normal group. Es $(165 \mathrm{mg} / 5 \mathrm{~mL} / \mathrm{kg}$ dissolved in distilled water) was administered orally by gavage once a day from the fourth week to sixth week in the Es group. DW was given at the same time and volume instead of Es in the normal and HFD groups.

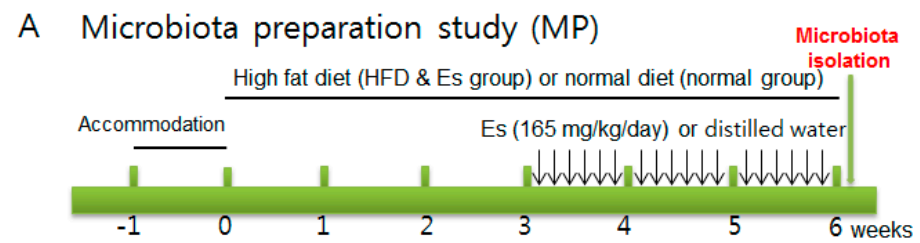

B Microbiota transplantation study (MT)
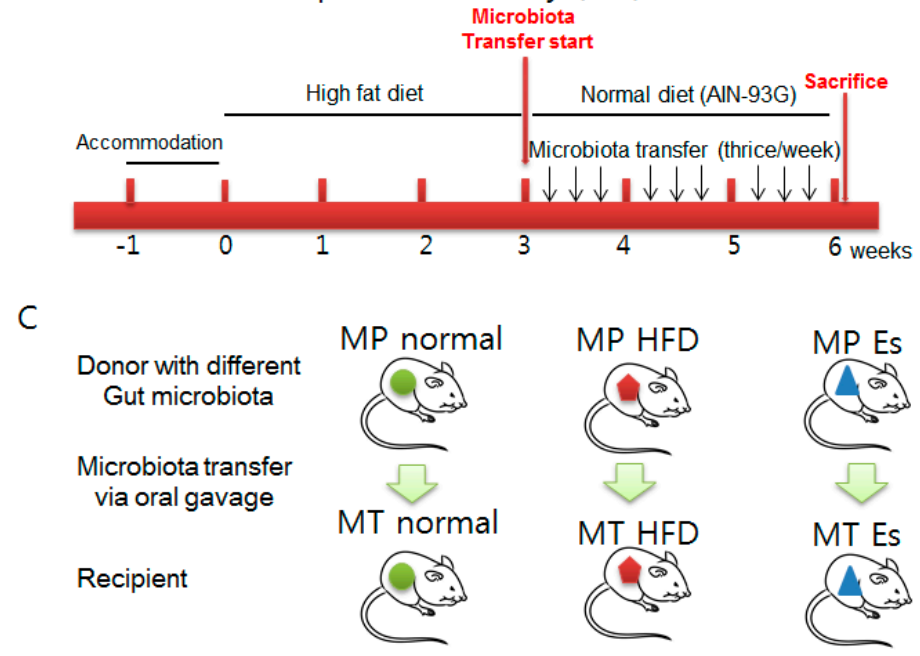

Figure 1. Experimental design microbiota preparation study (A) and microbiota transplantation study (B). The gut microbiota from microbiota preparation study were transplanted into its corresponding groups (C) as described in the Materials and Methods section. Abbreviations: MP, Microbiota preparation study; MT, Microbiota transplantation study; HFD, High-fat-diet; Es, Ephedra sinica. 
Table 1. Animal diets formulation and ingredients.

\begin{tabular}{ccccc}
\hline \multirow{2}{*}{ Formulation } & \multicolumn{2}{c}{ Normal Diet (AIN-93G) } & \multicolumn{2}{c}{ High Fat Diet } \\
\cline { 2 - 5 } & $\mathbf{g} \%$ & $\mathbf{k c a l} \%$ & $\mathbf{g} \%$ & $\mathbf{k c a l} \mathbf{0}$ \\
\hline Protein & 20 & 20 & 27 & 20 \\
Carbohydrate & 64 & 64 & 25 & 20 \\
Fat & 7 & 16 & 36 & 60 \\
kcal/kg & 4000 & & 5333 & \\
\hline Ingredient & $\mathbf{g}$ & $\mathbf{k c a l}$ & $\mathbf{g}$ & $\mathbf{k c a l}$ \\
\hline Casein (from milk) & 200 & 800 & 200 & 800 \\
Corn starch & 397,486 & 1590 & 47,536 & 190 \\
Sucrose & 100 & 400 & 0 & 0 \\
Dextrose & 132 & 528 & 132 & 528 \\
Cellulose & 50 & 0 & 50 & 0 \\
Soybean oil & 70 & 630 & 25 & 225 \\
Lard & 0 & 0 & 245 & 2205 \\
Mineral mixture & 35 & 0 & 35 & 0 \\
Vitamin mixture & 10 & 40 & 10 & 40 \\
TBHQ & 0.014 & 0 & 0.014 & 0 \\
L-Cystine & 3 & 12 & 3 & 12 \\
Choline bitartrate & 2.5 & 0 & 2.5 & 0 \\
Total & 1000 & 4000 & 750.1 & 4000 \\
\hline
\end{tabular}

Abbreviations: TBHQ, tertiary butylhydroquinone.

All animals were weighed, fecal samples were collected before and after the experiments, and stored at $-70{ }^{\circ} \mathrm{C}$. After six weeks, blood samples were collected from the abdominal aorta under anesthesia. Animals were sacrificed, the abdominal and epididymal fat pads and liver tissue were collected, and stored at $-70{ }^{\circ} \mathrm{C}$. The fresh cecal contents were separately collected and kept at $-80^{\circ} \mathrm{C}$ for transplanting. Before transplanting, all cecal components in each MP group were separately pooled and dissolved with ten times the volume of phosphate buffered saline (PBS).

The second step was the gut microbiota transplantation study (MT, Figure 1B): the additional nine male SD rats (6 weeks, 180-220 g, Orient Bio, Seongnam-si, Korea) were divided into three groups: MT normal, MT HFD, and MT Es group. First, feces were collected both before and after MT in all groups. HFD is a strong inducer of obesity and can markedly alter the gut bacterial ecosystem at both the structural and functional level. To evaluate anti-obesity, the HFD supply was stopped and changed to a normal diet before gut microbiota transplantation. Afterwards, MT HFD group animals $(n=3)$ received microbiota collected from the HFD group, MT Es group animals $(n=3)$ received microbiota obtained from Es treated group animals, and normal MT group animals $(n=3)$ received microbiota obtained from normal group animals. Prepared MP derived gut microbiota were administered by oral gavage $(10 \mathrm{~mL} / \mathrm{kg})$ three times per week for three weeks separately from the corresponding groups (Figure 1C). Eventually, the animals were weighed and sacrificed under anesthesia. Blood was collected from abdominal aorta and rapidly transferred into a BD Vacutainer (Franklin Lakes, NJ, USA). Subsequently, liver and fats were excised, weighed, rapidly dipped in physiological saline and stored at $-80^{\circ} \mathrm{C}$ for future use. After $2 \mathrm{~h}$ clotting, serum were separated by $3000 \times g$ centrifuge for $15 \mathrm{~min}$ and stored at $-20^{\circ} \mathrm{C}$ for future use. In both MP and MT, the volume of animal food intake was recorded by group. The Food Efficiency Ratio (FER) was computed by dividing the average body weight gain by the average food intake for each group.

\subsection{Biochemical Analyses}

Serum was separated using Vacutainer tubes (BD, Plymouth, UK) by $3000 \times g$ centrifuge for $15 \mathrm{~min}$ after blood clotting. The serum levels of aspartate transaminase (AST), alanine transaminase 
(ALT), fasting glucose, triglyceride (TG), total cholesterol (TC), and high density lipoprotein (HDL) were determined using an Auto Chemistry Analyzer (Hitachi, Tokyo, Japan).

\subsection{Enzyme Immunoassay for Serum Insulin}

Rat fasting serum insulin was measured using a Rat Insulin ELISA Kit (Mercodia, Uppsala, Sweden) according to the manufacturer's directions. Briefly, $10 \mu \mathrm{L}$ of samples or standards combined with $100 \mu \mathrm{L}$ of enzyme conjugate solution were added to an antibody coated plate, followed by incubation for two hours at room temperature. After washing six times with $700 \mu \mathrm{L}$ wash buffer solution per well, $200 \mu \mathrm{L}$ substrate TMB was added, followed by incubation for $15 \mathrm{~min}$ at room temperature. Finally, $50 \mu \mathrm{L}$ stop solution was added and the plate was read immediately on a spectrophotometer at $450 \mathrm{~nm}$. The concentration of fasting serum insulin was calculated by standard curve. The homeostatic model assessment for insulin resistance (HOMA-IR) is commonly used as an index of insulin resistance in clinic and epidemiological study. In the current study, HOMA-IR scores were calculated from fasting glucose and insulin levels according to the previous method [25].

\subsection{Fecal Acetic Acid Quantification by HPLC}

Frozen fecal samples were dissolved in HPLC-grade water (Sigma) at a proportion of $1 / 4$ $(w / v)$. After sonication for $10 \mathrm{~min}$ and $13,000 \times g$ centrifugation for $3 \mathrm{~min}, 0.2 \mu \mathrm{m}$ millipore filtered supernatants were stored at $-70{ }^{\circ} \mathrm{C}$. Prepared samples were analyzed using the YL9100 HPLC system (Young-Lin, Anyang, Korea) equipped with an Aminex-87H column (150 mm, $4.6 \mathrm{~mm}$, Bio-Rad, Hercules, CA, USA) and YL 9120 UV/Vis detector (Young-Lin). Acetic acid (Sigma-Aldrich) was used as a standard. Samples were eluted with $0.008 \mathrm{~N}$ sulfuric acid at $0.6 \mathrm{~mL} / \mathrm{min}$ for $30 \mathrm{~min}$. All chromatograms were obtained using a wavelength of $215 \mathrm{~nm}$ and YL-Clarity was applied for calculating concentrations of fecal organic acids according to the standard curve.

\subsection{Determination of Fecal Endotoxin}

The Limulus Amebocyte Lysate (LAL) test is an official method for endotoxin detection regulated by the US Food and Drug Administration [26]. The levels of fecal endotoxin were determined using a LAL kit (ENDOSAFE, Charleston, SC, USA) according to the manufacturer's protocol. PBS dissolved fecal supernatant samples were added into $5 \mathrm{EU} / \mathrm{mL}$ standard spiked or non-spiked wells in duplicate. Following addition of $100 \mu \mathrm{L}$ LAL reagent, the kinetic absorbance of the mixture was measured at $405 \mathrm{~nm}$ and the reaction onset times of the samples were compared to the standard curve. The quality of data was confirmed in accordance with the spike recovery rates within $50 \%$ to $200 \%$.

\subsection{Real-Time Polymerase Chain Reaction (PCR)}

Real-time PCR was used to determine the MCP-1, IL-1 $\beta$ and $\beta$-actin gene expression in liver tissue using following primers (MCP-1, forward 5'-CAG CCA GAT GCA GTT AAT GCC-3', reverse 5'-AGC CGA CTC ATT GGG ATC AT-3'; IL-1 $\beta$, forward 5'-CAC CTC TCA AGC AGA GCA CAG-3', reverse $5^{\prime}$-GGG TTC CAT GGT GAA GTC AAC- $3^{\prime}$; and $\beta$-actin, forward $5^{\prime}$-AGA GGG AAA TCG TGC GTG AC-3', reverse 5'-CCA TAG TGA TGA CCT GTC CGT-3'). Total RNA was isolated using TRIsure reagent (Bioline, Taunton, MA, USA), followed by cDNA synthesis using the AccuPower RT premix kit (Bioneer, Daejeon, Korea). PCR was performed using a LightCycler FastStart DNA Master SYBR Green kit and a LightCycler instrument (Roche, Indianapolis, IN, USA). The standard conditions for the PCR amplification reactions were applied, as previously described [17]. The LightCycler software (Roche Applied Science) was used for analysis. Relative gene expression was represented by $2^{-\Delta c t}$ calculations $\left(\Delta \mathrm{Ct}=\mathrm{Ct}_{\text {target gene }}-\mathrm{Ct}_{\beta \text {-actin }}\right)$. The results are expressed as normalized fold values relative to the normal group. 


\subsection{Pyrosequencing and Bioinformatics Analysis}

Fecal samples were collected and immediately stored at $-70^{\circ} \mathrm{C}$. Genomic DNA was extracted using the QIAamp DNA feces mini kit (Qiagen, Hilden, Germany). Two or three samples from each group were selected for the pyrosequencing analysis. Pyrosequencing analyses were performed at Chun Lab (Seoul, Korea). The target region of the 16S rRNA genes (V1-V3 of a variable region was amplified using a C1000 Touch thermal cycler (Bio-Rad, Hercules, CA, USA) and purified using a QIAquick PCR purification kit (Qiagen). After quantification of purified products using the PicoGreends DNA Assay kit (Invitrogen, Carlsbad, CA, USA), equimolar concentrations of each amplicon were sequenced on a Roche/454 GS Junior system according to the manufacturer's instructions. For analysis of pyrosequencing data, raw sequences for each sample were sorted according to their unique barcodes. Low quality reads (average quality scores $<25$ or read length $<300 \mathrm{bps}$ ) were filtered and primer sequences were trimmed using pair-wise alignment and HMMER 3.0 package (HHMI Janelia Farm Research Campus, Ashburn, VA, USA). Trimmed sequences were corrected for sequencing errors by clustering, and representative sequences in each cluster were assigned their taxonomic position. Taxonomic identification was performed using the EzTaxon-e database according to the highest pair wise similarity among the top five BLASTN (Basic Local Alignment Search Tool Nucleotide) results. Possible chimera sequences were removed using the UCHIME. Operational taxonomic units picking, taxonomic assignment and phylogentic reconstruction were analyzed using QIIME (Version 1.9.1, University of Colorado, Boulder, CO, USA) software package and visualization in R Programming (Version 3.2.2, University of Auckland, Auckland, New Zealand). The functional and taxonomic difference of metagenomics data were predicted by PICRUSt-1.0.0 (Phylogenetic Investigation of Communities by Reconstruction of Unobserved States) [27] and HUMAnN2 (The HMP Unified Metabolic Analysis Network 2, http:/ / huttenhower.sph.harvard.edu/humann2) [28]. The significant predicted results were statistically analyzed and represented graphically by STAMP v2.1.3 software (Dalhousie University, Halifax, Canada, http://kiwi.cs.dal.ca/Software/STAMP).

\subsection{Statistical Analysis}

All values were analyzed using one-way ANOVA followed by LSD (least significant difference) post-hoc test using the Statistical Package for Social Science (release 2008, SPSS Statistics for Windows, Version 17.0. SPSS Inc., Chicago, IL, USA) software. The results were expressed as the mean \pm standard deviation (SD). Null hypotheses of no difference were rejected if $p$-values were less than 0.05 .

\section{Results}

\subsection{Es Extraction Yield and Ephedrine Proportion}

The final extraction of Es gave a yield of $14.83 \%(w / w)$. By calculating with a standard curve $\left(\mathrm{R}^{2}=1\right.$, data not shown), the proportion of ephedrine in Es extract was approximately equal to $1.798 \%$ $(w / w)$ (Figure 2A,B). Therefore, $29.7 \mathrm{mg} / \mathrm{kg}$ of ephedrine was administered in the MP study, while ephedrine was not detected in MP Es feces according to the HPLC results (Figure 2C-F). 
A

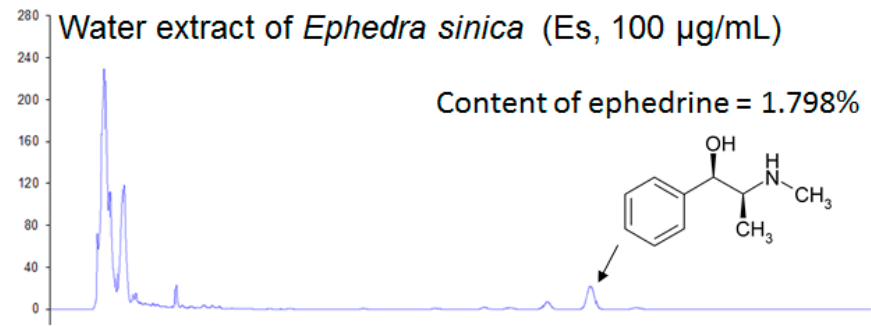

B

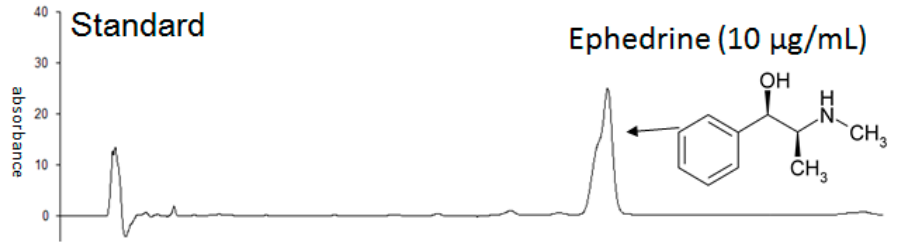

C

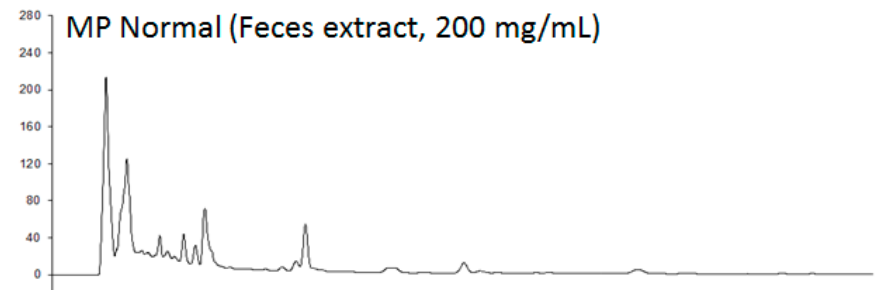

D

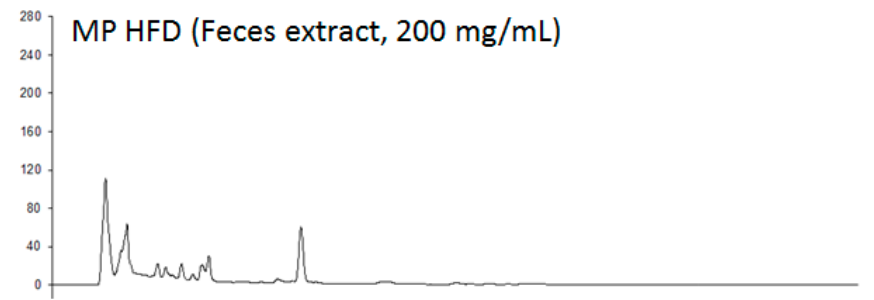

E

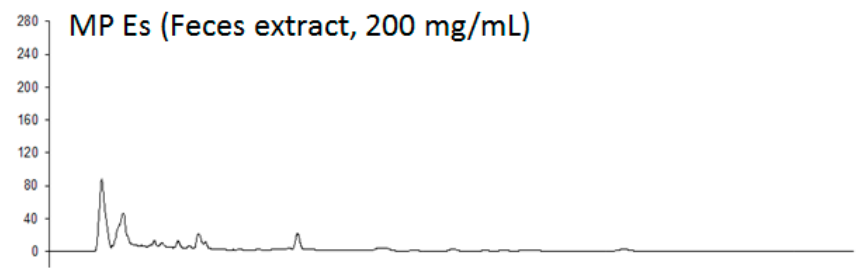

F

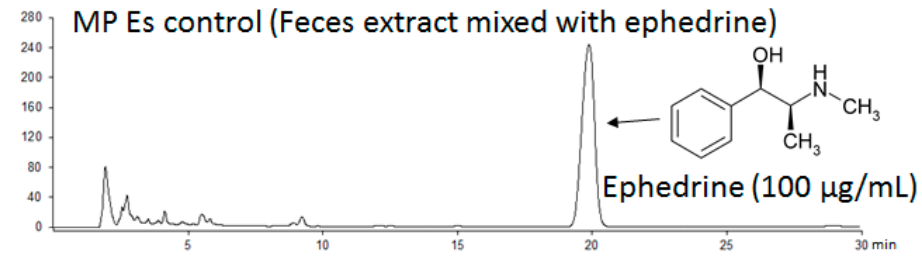

Figure 2. High-performance liquid chromatography (HPLC) fingerprinting of the water extract of Ephedra sinica (Es) and standards were filtered and subjected to HPLC analysis. (A) Water extract of Es $(100 \mu \mathrm{g} / \mathrm{mL}),(\mathbf{B})$ standard: ephedrine $(10 \mu \mathrm{g} / \mathrm{mL}),(\mathbf{C}) \mathrm{MP}$ normal fecal extract $(200 \mathrm{mg} / \mathrm{mL}),(\mathbf{D}) \mathrm{MP}$ HFD fecal extract (200 mg/mL), (E) MP Es fecal extract $(200 \mathrm{mg} / \mathrm{mL})$, (F) MP Es control (MP Es fecal extract mixed with $100 \mu \mathrm{g} / \mathrm{mL}$ of ephedrine). The chromatograms were produced using a wavelength of $215 \mathrm{~nm}$.

3.2. Both Es and Es Altered Gut Microbiota Changed Body Weight (Gain), Fats and Organs Weight and Food Intake

Es treatment significantly reduced body weight (gain), and food intake compared to the MP HFD group. EsM showed the same pattern in accordance with Es extract. Both Es treatment and EsM significantly decreased the food intake compared to the corresponding HFD group. HFD caused a 
marked increase in fat weight, including abdominal fat, epididymal fat, and perirenal fat, compared to the MP normal group. Es treatment resulted in significantly decreased body weight, and body weight gain compared to the MP HFD group. EsM showed the same pattern in accordance with Es extract. Both Es extract and EsM influenced appetite compared to the corresponding HFD group (Table 2). Consequently, both HFD and HFD alteration of gut microbiota resulted in significantly increased FER compared to the corresponding normal group; however, Es and EsM did not change the HFD elevated FER.

Table 2. Comparison of body, fat and organ weights, food intake and food-efficiency ratio.

\begin{tabular}{|c|c|c|c|c|c|c|}
\hline \multirow[b]{2}{*}{ Groups } & \multicolumn{3}{|c|}{ Microbiota Preparation Experiment (MP) } & \multicolumn{3}{|c|}{ Microbiota Transfer Experiment (MT) } \\
\hline & MP Normal & MP HFD & MP Es & MT Normal & MT HFD & MT Es \\
\hline Initial body weight (g) & $244.0 \pm 9.6$ & $248.0 \pm 7.7$ & $244.9 \pm 10.2$ & $271.1 \pm 3.3$ & $268.2 \pm 4.9$ & $272.9 \pm 6.0$ \\
\hline Final body weight (g) & $444.9 \pm 20.3$ & $553.6 \pm 61.7^{\#}$ & $487.9 \pm 56.8^{*}$ & $524.6 \pm 21.5$ & $587.3 \pm 5.6^{\&}$ & $532.8 \pm 14.1^{\$}$ \\
\hline Body weight gain $(g)$ & $200.9 \pm 23.8$ & $305.6 \pm 59.9^{\#}$ & $249.1 \pm 42.9$ * & $253.5 \pm 18.2$ & $319.1 \pm 10.3^{\&}$ & $260.0 \pm 12.0^{\$}$ \\
\hline Food intake (g/day) & $23.8 \pm 2.5$ & $22.0 \pm 2.8$ & $18.1 \pm 3.7 *$ & $25.3 \pm 0.8$ & $22.1 \pm 0.8^{\&}$ & $18.7 \pm 1.2^{\$}$ \\
\hline Food efficiency ratio & $0.21 \pm 0.02$ & $0.35 \pm 0.08^{\#}$ & $0.36 \pm 0.11$ & $0.24 \pm 0.01$ & $0.34 \pm 0.00^{\&}$ & $0.33 \pm 0.01$ \\
\hline Total fat (g) & $46.0 \pm 4.9$ & $82.3 \pm 19.2^{\#}$ & $63.1 \pm 6.7^{*}$ & $52.5 \pm 9.8$ & $83.1 \pm 5.9^{\&}$ & $58.5 \pm 2.8^{\$}$ \\
\hline Relative of total fat (\%) & $7.89 \pm 0.66$ & $11.75 \pm 2.90^{\#}$ & $10.06 \pm 0.73$ & $10.0 \pm 1.5$ & $14.2 \pm 1.1 \&$ & $10.9 \pm 0.4^{\$}$ \\
\hline Abdominal fat (g) & $10.8 \pm 1.4$ & $17.9 \pm 5.1^{\#}$ & $13.1 \pm 2.3^{*}$ & $11.3 \pm 4.1$ & $17.1 \pm 1.4$ & $16.3 \pm 2.0$ \\
\hline Epididymal fat (g) & $9.8 \pm 0.9$ & $17.8 \pm 3.9^{\#}$ & $13.6 \pm 2.1 *$ & $17.3 \pm 2.4$ & $28.0 \pm 1.8^{\&}$ & $15.0 \pm 1.2^{\$}$ \\
\hline Perirenal fat (g) & $14.5 \pm 2.4$ & $28.6 \pm 5.9^{\#}$ & $22.5 \pm 3.4$ & $23.9 \pm 4.0$ & $38.0 \pm 4.1^{\&}$ & $27.2 \pm 4.5^{\$}$ \\
\hline Liver weight (g) & $12.9 \pm 1.5$ & $16.4 \pm 2.1^{\#}$ & $14.1 \pm 2.3$ & $15.0 \pm 0.8$ & $15.2 \pm 0.7$ & $15.5 \pm 1.6$ \\
\hline Relative of Liver weight (\%) & $2.91 \pm 0.34$ & $3.00 \pm 0.56$ & $2.88 \pm 0.26$ & $2.9 \pm 0.1$ & $2.6 \pm 0.1^{\&}$ & $2.9 \pm 0.3$ \\
\hline Cecum weight $(\mathrm{g})$ & $5.6 \pm 0.8$ & $4.6 \pm 0.2^{\#}$ & $5.0 \pm 0.3^{*}$ & $4.2 \pm 0.1$ & $4.6 \pm 0.1 \&$ & $5.3 \pm 1.3$ \\
\hline Relative of Cecum weight (\%) & $1.26 \pm 0.2$ & $0.84 \pm 0.08^{\#}$ & $1.04 \pm 0.09 *$ & $0.80 \pm 0.04$ & $0.79 \pm 0.02$ & $0.99 \pm 0.27$ \\
\hline Cecum contents $(\mathrm{g})$ & $1.90 \pm 0.57$ & $1.39 \pm 0.19^{\#}$ & $1.69 \pm 0.21 *$ & $1.7 \pm 0.2$ & $1.1 \pm 0.1 \&$ & $1.5 \pm 0.2^{\$}$ \\
\hline
\end{tabular}

Data are expressed as mean \pm SD and statistically evaluated using one way ANOVA followed by Least Significant Difference (LSD) pos-hoc test. ${ }^{\#} p<0.05$, compared to the MP normal group; ${ }^{*} p<0.05$ compared to the MP HFD group $(n=8) ;{ }^{\&} p<0.05$, compared to the normal MT group; $\$ p<0.05$ compared to the MT HFD group $(n=3)$. Relative tissues weight $(\%)=($ tissue weight $/$ final body weight $) \times 100 \%$. Food efficiency ratio $=$ body weight gain (g/day)/food intake (g/day).

\subsection{EsM Ameliorated Hyperglycemia and Hyperinsulinemia, but Did Not Alter Serum Aminotransferases and} Lipid Parameters

Except that HFD obviously elevated the serum ALT compared to the MP normal group, both Es and EsM did not notably change the level of serum AST and ALT. Similarly, HFD feed for six weeks significantly increased serum TG level compared to the normal diet, while Es treatment resulted in noticeably reduced serum TG level compared to the MP HFD group. However, in microbiota transplantation groups, the serum TG and TC level was not noticeably altered. HFD induced a prominent increase in FBG, serum insulin, and HOMA-IR compared to normal diet. Both Es and EsM resulted in markedly reduced FBG, serum insulin, and HOMA-IR (Table 3).

Table 3. Comparison of serum biochemical parameters.

\begin{tabular}{|c|c|c|c|c|c|c|}
\hline \multirow[b]{2}{*}{ Groups } & \multicolumn{3}{|c|}{ Microbiota Preparation Experiment (MP) } & \multicolumn{3}{|c|}{ Microbiota Transfer Experiment (MT) } \\
\hline & MP Normal & MP HFD & MP Es & MT Normal & MT HFD & MT Es \\
\hline AST (IU/L) & $76.8 \pm 10.8$ & $73.9 \pm 13.7$ & $80.4 \pm 14.1$ & $79 \pm 7.8$ & $151.3 \pm 41.2$ & $113.0 \pm 26.0$ \\
\hline ALT (IU/L) & $23.3 \pm 4.2$ & $35.4 \pm 5.6^{\#}$ & $35.1 \pm 10.3$ & $30.0 \pm 6.2$ & $32.3 \pm 2.9$ & $31.7 \pm 2.5$ \\
\hline $\mathrm{TG}(\mathrm{mg} / \mathrm{dL})$ & $28.8 \pm 1.3$ & $59.0 \pm 12.1^{\#}$ & $43.2 \pm 6.1 *$ & $43.0 \pm 6.1$ & $54.0 \pm 11.4$ & $39.7 \pm 13.6$ \\
\hline $\mathrm{TC}(\mathrm{mg} / \mathrm{dL})$ & $47.9 \pm 7.8$ & $52.9 \pm 5.3$ & $59.3 \pm 11.9$ & $65.7 \pm 7.4$ & $66.0 \pm 4.6$ & $59.3 \pm 9.5$ \\
\hline HDL (mg/dL) & $28.6 \pm 11.2$ & $24.3 \pm 2.0$ & $33.1 \pm 4.3^{*}$ & $35.2 \pm 4.1$ & $29.7 \pm 4.6$ & $28.7 \pm 5.3$ \\
\hline $\mathrm{FBG}(\mathrm{mg} / \mathrm{dL})$ & $135.8 \pm 27.5$ & $186.4 \pm 29.8^{\#}$ & $145.1 \pm 25.6 *$ & $158.7 \pm 18.5$ & $268.7 \pm 12.9 \&$ & $190.0 \pm 21.4^{\$}$ \\
\hline Insulin (ng/mL) & $0.28 \pm 0.13$ & $0.65 \pm 0.14^{\#}$ & $0.52 \pm 0.07 *$ & $0.24 \pm 0.15$ & $0.57 \pm 0.11 \&$ & $0.28 \pm 0.08^{\$}$ \\
\hline HOMA-IR (mg/dL) & $0.09 \pm 0.04$ & $0.29 \pm 0.05^{\#}$ & $0.18 \pm 0.04 *$ & $0.11 \pm 0.02$ & $0.38 \pm 0.09 \&$ & $0.11 \pm 0.06^{\$}$ \\
\hline
\end{tabular}

Abbreviations: AST, aspartate transaminase; ALT, alanine transaminase; TC, total cholesterol; HDL, high density lipoprotein; TG, triglyceride; FBG, fasting blood-glucose. Data were expressed as mean \pm SD and statistically evaluated using one-way ANOVA followed by LSD post-hoc test. ${ }^{\#} p<0.05$ compared to the MP normal group; ${ }^{*} p<0.05$ compared to the MP HFD group $(n=8)$; ${ }^{\&} p<0.05$ compared to the MT normal group; ${ }^{\$} p<0.05$ compared to the MT HFD control group $(n=3)$. 


\subsection{Both Es and EsM Ameliorated Enterogenous Endotoxin}

HFD and HFD alteration of gut microbiota identically induced a significant increase in fecal endotoxin level compared to the corresponding normal group, while Es and EsM also identically resulted in evident diminution of the fecal endotoxin level compared to the corresponding HFD group (Figure 3A).
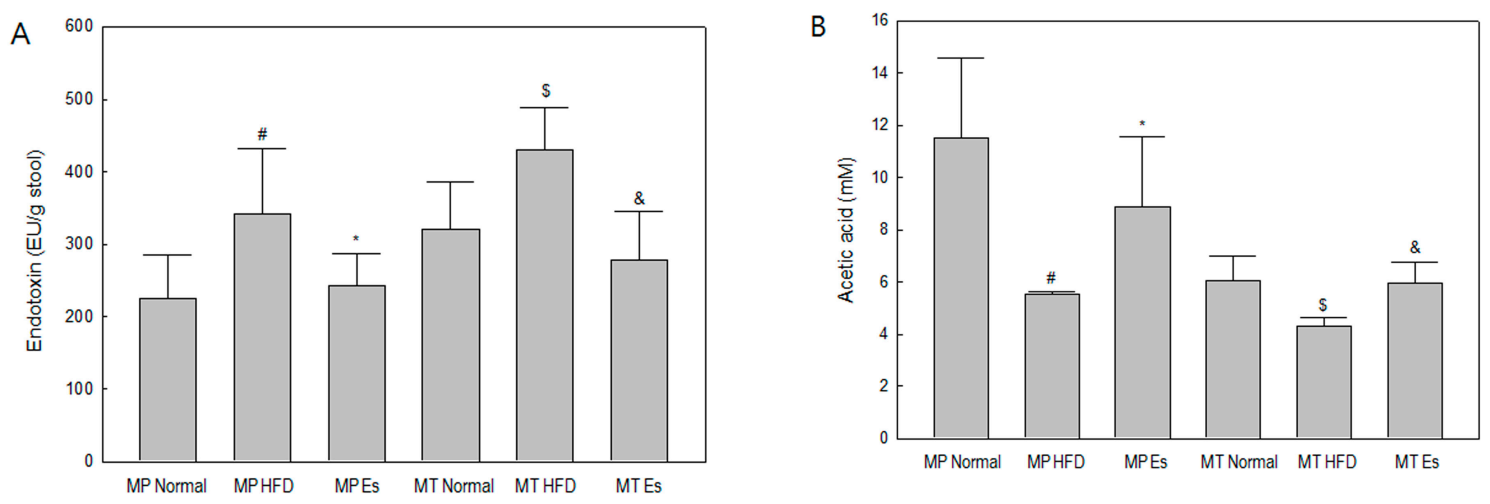

Figure 3. Quantitative determination of endotoxin and Acetic acid level in rat feces at the end of experimental schedule, the stool was collected from the animals. (A) The fecal endotoxin level was determined using a Limulus Amebocyte Lysate (LAL) kit. Data are expressed as mean $\pm \mathrm{SD}$ (standard deviation). ${ }^{\#} p<0.05$ compared to the MP normal group; ${ }^{*} p<0.05$ compared to the MP HFD group $(n=8) ;{ }^{\$} p<0.05$ compared to the MT normal group; ${ }^{\&} p<0.05$ compared to the MT HFD group $(n=3)$;

(B) The fecal acetic acid level was determined using the YL9100 HPLC system and an Aminex-87H column. HPLC chromatograms were obtained at a wavelength of $215 \mathrm{~nm}$. Level of fecal acetic acids were calculated by YL-Clarity according to the standard curve.

\subsection{Both Es and EsM Elevated the Fecal Acetic Acid Level}

HFD and HFD alteration of gut microbiota similarly reduced the acetic acid level in feces compared to the corresponding normal group. Both Es and EsM also led to marked restoration of the fecal acetic acid level compared to the corresponding HFD group (Figure 3B).

\subsection{Both Es and EsM Down-Regulated Gene Expression of Key Proinflammatory Cytokines}

HFD and HFD alteration of gut microbiota similarly resulted in significant up-regulation of the gene expression of MCP- 1 and IL-1 $\beta$ compared to the corresponding normal group. Both Es and EsM resulted in down-regulation of $M C P-1$ gene expression compared to the corresponding HFD group. Only EsM, not Es itself, significantly down-regulated gene expression of IL- $1 \beta$ compared to the corresponding HFD group (Figure 4A,B). 
A

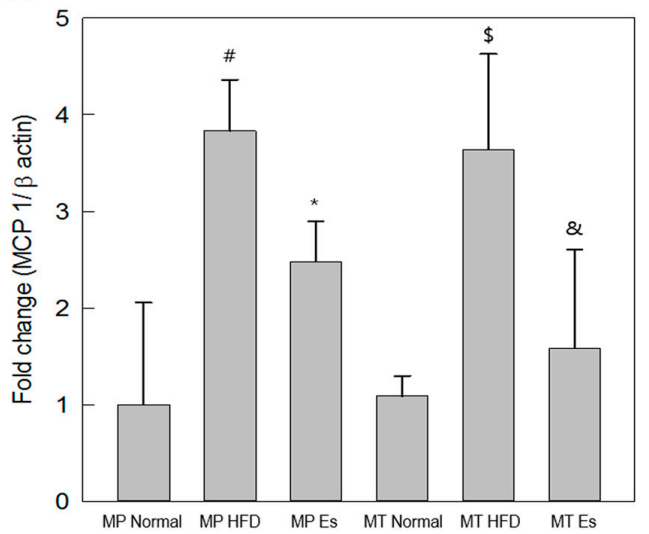

B

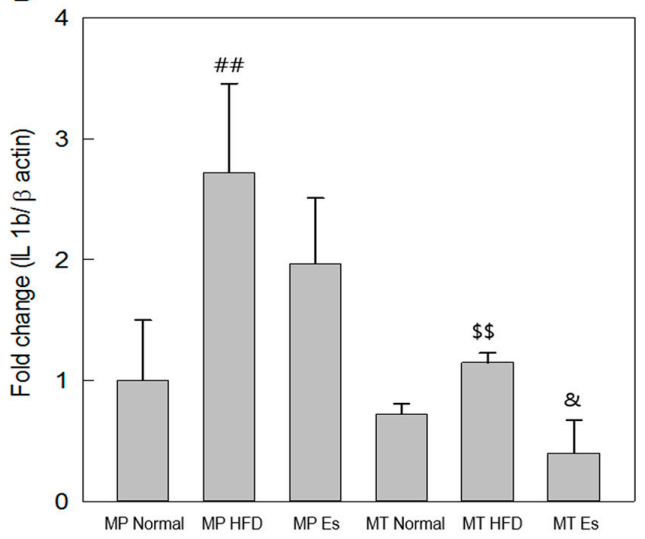

Figure 4. Real-time polymerase chain reaction (PCR) analysis. The gene expression of the MCP-1 (A) and IL-1 $\beta$ (B) were analyzed by real-time PCR in liver tissue. The results are expressed as normalized fold values relative to the MP normal group. ${ }^{\#} p<0.05,{ }^{\#} p<0.01$ compared to the MP normal group; ${ }^{*} p<0.05$ compared to the MP HFD group $(n=8) ;{ }^{\$} p<0.05,{ }^{\$} p<0.01$ compared to the MT normal group; \& $p<0.05$ compared to the MT HFD group $(n=3)$.

\subsection{Gut Microbiota from Donor Evidently Influenced the Host Gut Microbial Community}

More than $0.5 \%$ of family and genus in donor and recipient were selected for gut microbiota transplantation comparability checking (Figure 5). At family level, 61.54\%, 84.62\%, and 71.43\% of donors were in accordance with their corresponding receptor. Similarly, $45.71 \%, 70.27 \%$, and $75.68 \%$ of donors were in accordance with their corresponding recipient at genus level (Figure S1).

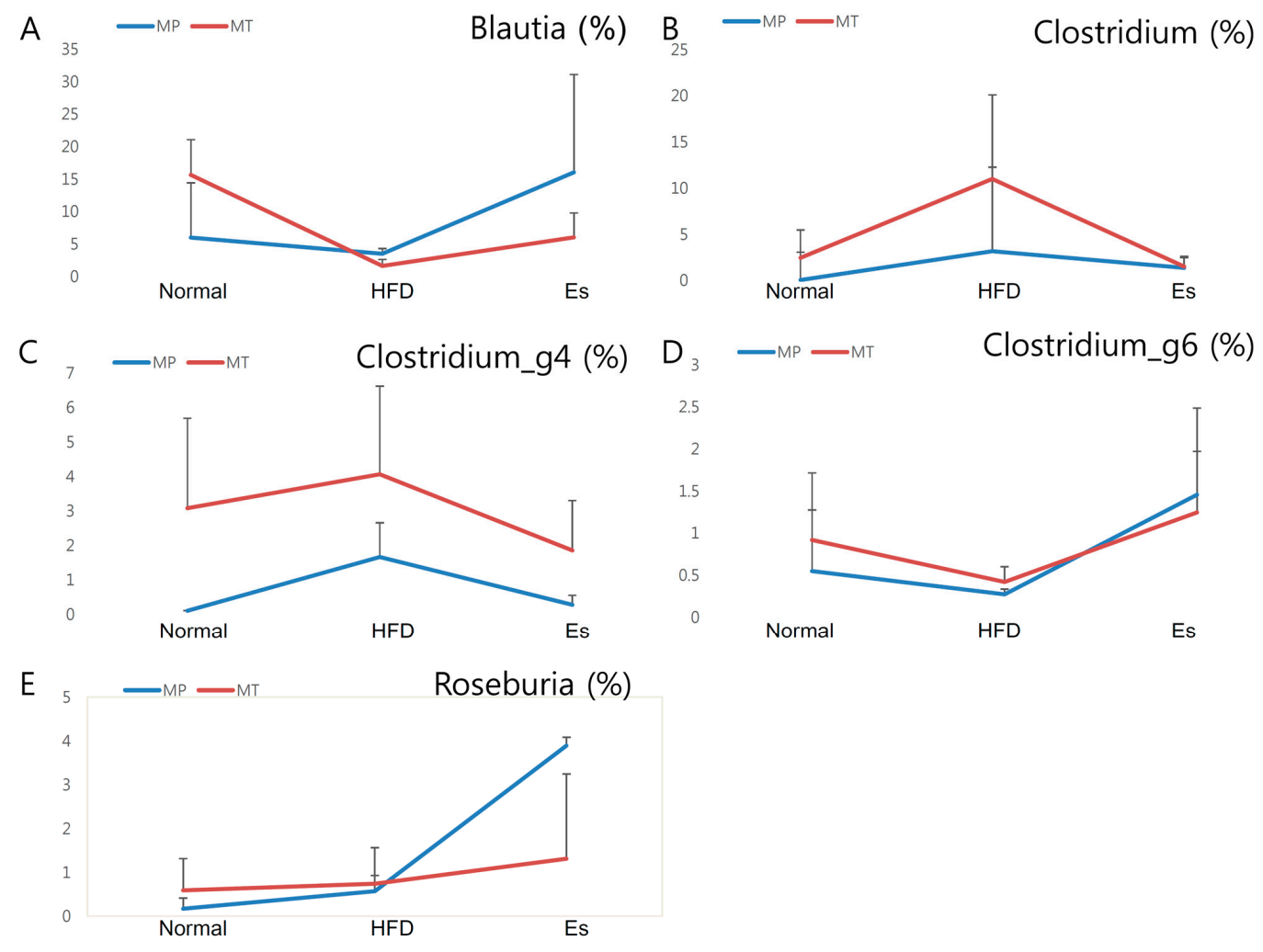

Figure 5. Cont. 
F
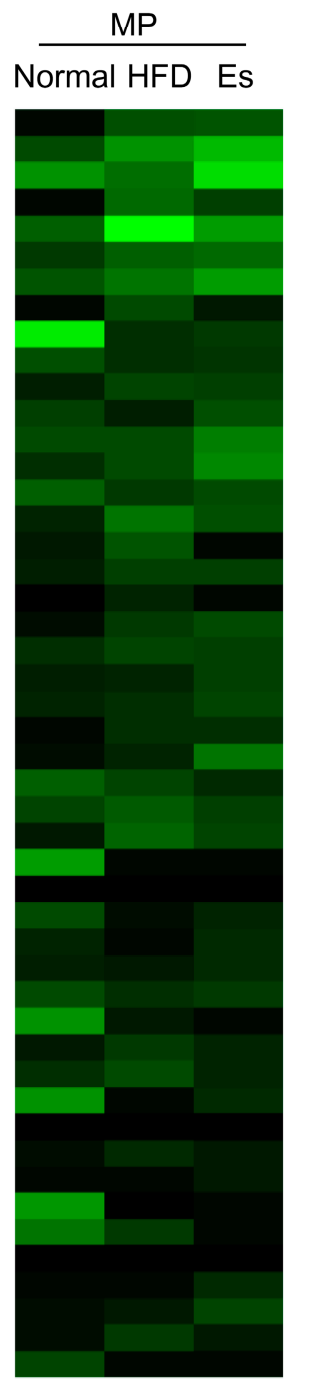

The colors scale

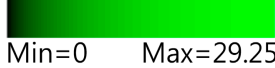

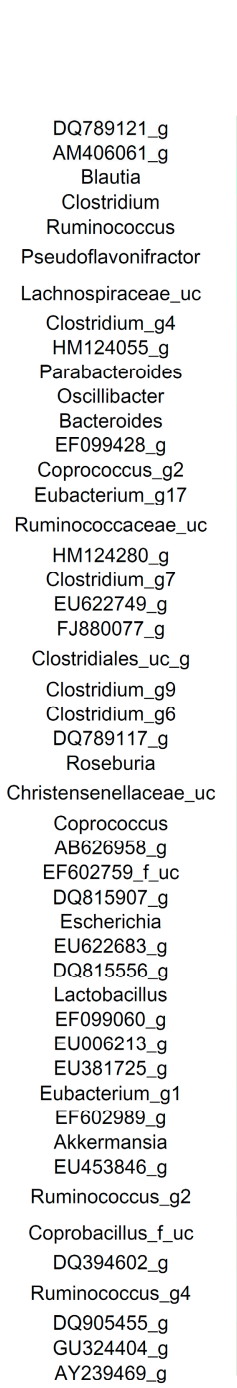

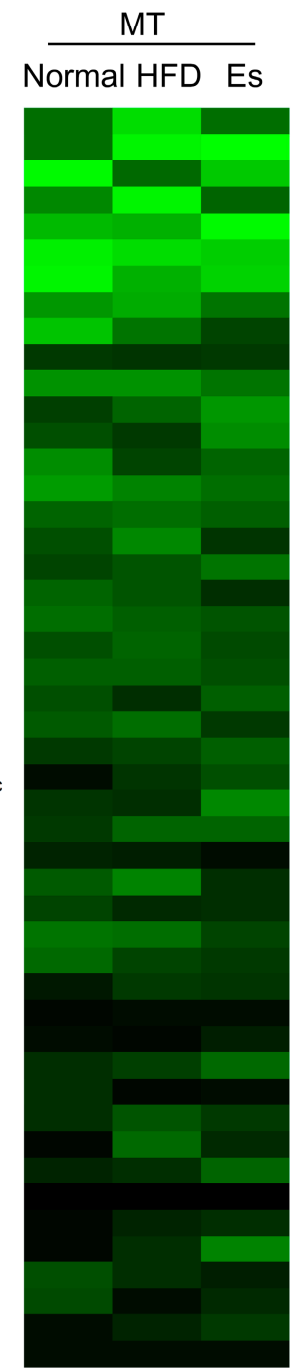

The colors scale

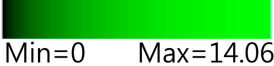

Figure 5. Comparison of genus level of gut microbiota At least more than $0.5 \%$ of genus of gut microbiota in any group were selected according to the pyrosequencing data. The variation tendency of several representative genera, like Blautia (A); Clostridium (B); Clostridium_g4 (C); Clostridium_g6 (D) and Roseburia (E), showed a conformity pattern between MP and MT experiment; (F) At least more than $1 \%$ of genus in any group were selected and compared using heatmap. The visualized heatmap were figured by PermutMatrix (Version 1.9.3 EN). As the colors scale shown, green color indicates a higher proportion, while black color shows a lower proportion.

\subsection{Predicted Alteration of Gut Microbiome Function}

PICRUSt and HUMAnN2 pipelines were applied to predictive functional and taxonomic profiling of gut microbial communities using 16s rRNA gene sequences. HFD altered gut microbiota markedly increased the peroxisome and decreased the glycolysis/gluconeogenesis as compared to MT normal group. However, EsM remarkably suppressed these changes compared to MT HFD group (Figure 6A,B). The functional pathway predicted results showed that EsM significantly changes the lipopolysaccharide biosysnthesis pathway (ko00540) and glycolysis/gluconeogenesis pathway (ko00010) compared to MT HFD group (Figure 6C,D). 
A

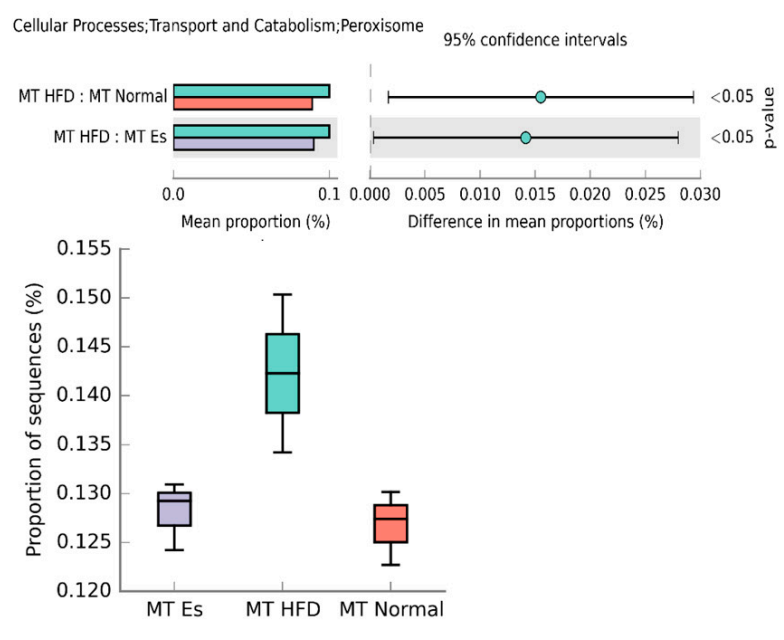

C ko00540: Lipopolysaccharide_ biosynthesis

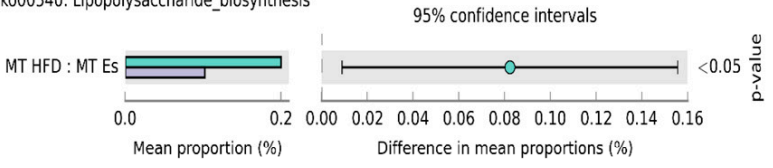

Mean proportion (\%) Difference in mean proportions (\%)

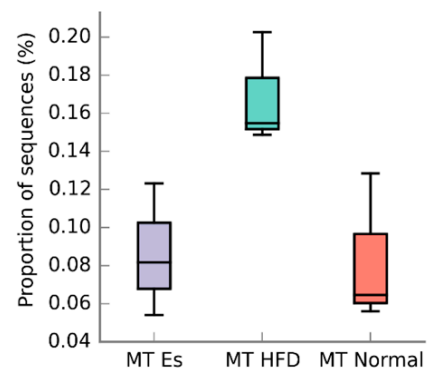

Metabolism;Carbohydrate Metabolism;Glycolysis / Gluconeogenesis $95 \%$ confidence intervals

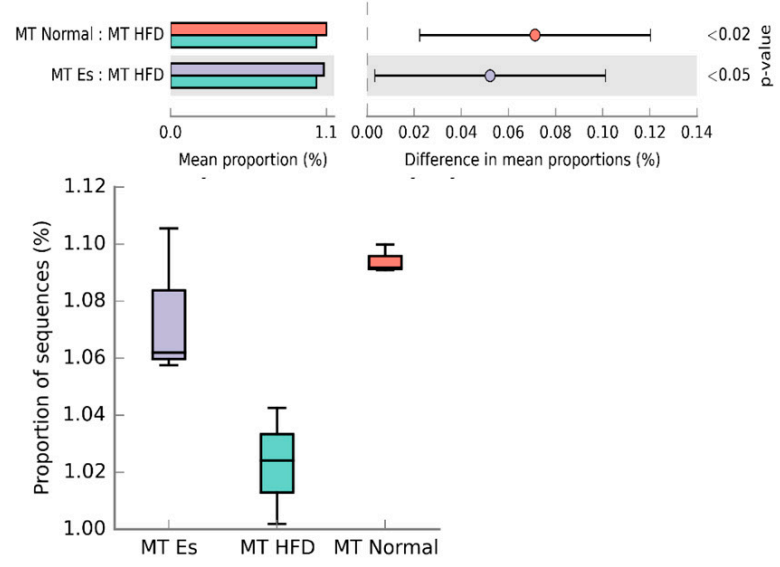

D ko00010: Glycolysis_Gluconeogenesis

$95 \%$ confidence intervals
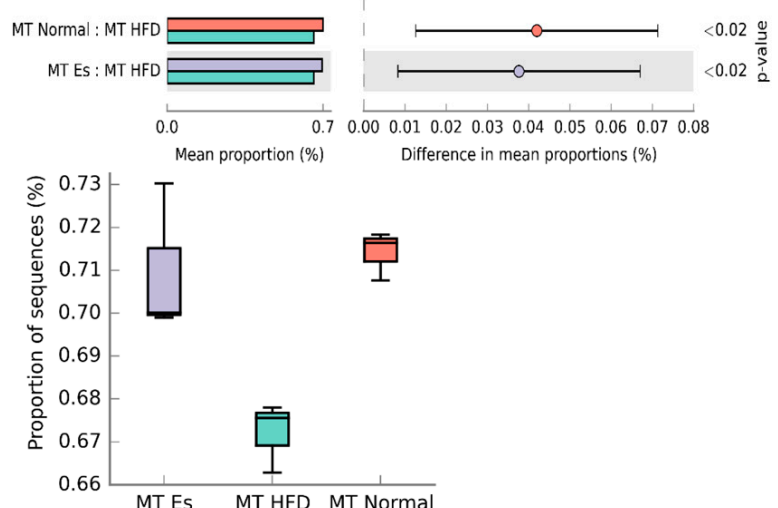

Figure 6. Predicted difference of gut microbiome function and KEGG pathway. The significant difference $(p<0.05$, confidence intervals equal 95\%) of PICRUSt (A,B) and HUMAnN2 (C,D) derived predictive finding was figured by STAMP (Version 2.1.3). 


\section{Discussion}

In both developed and developing countries, obesity has been rapidly increasing for the past 30 years [29]. More than 2.1 billion people (approximately one third the world's population) are overweight and obese worldwide until 2013 [29]. Unhealthful dietary habits, like high fat diet, directly change the normal distribution of gut microbiota following the reduction of intestinal microbiota diversity [13,30]. Dysfunction of gut microbiota evidently promotes development of chronic metabolic disorders [31] via regulation of energy homeostasis and fat storage [32]. Therefore, modulating intestinal microbiota was deemed a feasible strategy for treatment of obesity and related diseases. Our previous studies verified that medicinal herbs effectively ameliorate obesity and metabolic endotoxemia associated with regulation of gut microbiota distribution and gut permeability in an animal metabolic disorder model $[17,33]$.

HFD alters gut microbiota, and can directly cause obesity independent of dietary type in a gnotobiotic mouse model [34]. Es exerted a beneficial effect on obesity and glucose intolerance in a HFD mice model [7]. Our previous study demonstrated that although the individual sensitivity to ephedra differs, alteration of gut microbiota by Ephedra intake was associated with loss of BW and BMI in human subjects [24]. In the MP, three types of gut microbiota (from normal, HFD, or HFD together with Es treatment, respectively) were transplanted by oral gavage to 3-week HFD fed rats. It is noteworthy that ephedrine was not detected even in Es treatment fecal samples and there was no obvious difference among the three types of MP feces extract according to the HPLC profiling. Thereby it is sufficient to exclude the transplantation effect caused by ephedrine. Though the antibiotics can exceedingly disrupt intestinal microbial ecology, it seems more facile to transplant gut microbiota from donor, however none scientific evidence revealed antibiotics pretreatment before fecal transplantation is more beneficial than non-treatment in duplication of donor phenotypes and bacterial phylotypes, therefore in the current study we did not use any antibiotics before transplantation to eliminate the preexisting gut bacteria. The conventional recipient is more similar to a clinical obesity model than a gnotobiotic model with physiological abnormalities. As expected, Es administration obviously reduced the body weight (gain), total visceral weight and food intake compared to the HFD control group. Commonly Es is considered an appetite suppressant for weight loss due to its Ephedra alkaloid compounds. Notably our data showed that EsM transplantation reduces body weight, total visceral weight and intake of food, without interfering with FER. The above results indicate that appetite suppression by EsM transplantation is a potential anti-obesity mechanism.

A short-chain fatty acid acetate that can directly reduce appetite via regulation of central homeostasis was reported [35]. Many gut bacterial species, including Lactobacillus spp., can promote acetate production in HFD-fed mice [36], and acetate suppresses body fat accumulation in rats [37]. High levels of fecal acetic acid were observed in both the Es treatment group and EsM transplantation group. This indicates that EsM maintained the body weight and visceral fat weight loss effect through augmentation of the short chain fatty acid acetate, bringing about the anorexigenic property.

In addition, to investigate the change of visceral fat in detail, the weights of mesenteric, epididymal, and perirenal fat were compared separately. Interestingly, Es administration resulted in noticeably decreased mesenteric and epididymal fat weight compared to HFD control. However, EsM more obviously decreased epididymal fat and perirenal fat weight. Es and EsM showed a partial difference in characteristics of visceral fat distribution. Es administration did not reduce the weight of fat liver increased by HFD. Both HFD altered gut microbiota (HFM) and EsM did not influence liver weight. Serum aminotransferases also did not change in any microbiota transplantation groups. Although the microbiota alteration influencing lipid and glucose metabolism has been demonstrated in an ob/ob mice model [38], in our experiment alteration of gut microbiota transplantation resulted in amelioration of fasting serum glucose but not lipid profile, such as TG, TC, and HDL. This might be due to a short period (three weeks) of microbiota transplantation and individual differences of SD rats. Antibiotic alteration of gut microbiota improves insulin resistance in diet-induced obesity [39]. In the current experiments, both Es and EsM transplantation resulted in improvement of insulin 
resistance, as evidenced by inhibition of serum insulin concentration and HOMA-IR. Besides, EsM significantly enhanced glycolysis and gluconeogenesis through influence of metabolic pathway (ko00010) (Figure 6B,D). It is probable evidence for improving hyperglycemia and hyperinsulinemia associated with gut microbiota change.

HFD significantly affects the gut microbiota ecosystem at the functional level [30]. HFD feeding decreased the weight of cecum contents via alteration of intestinal bacteria metabolism while antibiotic treatment significantly increased it [13]. In the current experiment, both Es and EsM resulted in markedly augmented HFD lowered weight of cecum contents. The effect of the cecal weight by Es or ESM might involve a close association with the impact of microbiota diversity and composition. The PCoA analysis did not show an obviously different pattern of gut microbiota composition in each group (Figure S2). However, family and genus level of gut microbiota were similar between donor and recipient (Figure 5, Figure 7 and Figure S1).

Low-grade inflammation triggered by gut microbiota-derived endotoxin, leading to initiation of obesity and insulin resistance was confirmed [12]. HFD induced significant elevation of the fecal endotoxin although Es notably prevented the increase. It is noteworthy that gut microbiota transplantation groups showed a similar pattern of fecal endotoxin compared to each donor. It might be that the pattern of gut microbiota is closely associated with the intestinal endotoxin environment. In accordance with fecal endotoxin pattern, KEGG pathway predictions from HUMAnN2 based on 16s rRNA bacteria gene sequences indicated that EsM significantly negatively impact on LPS biosynthesis pathway (ko00540). Enteral endotoxin induced inflammation was evidenced by high expression of IL-1 beta and MCP-1 cytokines. As demonstrated, IL-1 $\beta$ is a quintessential pro-inflammatory cytokine associated with obesity and metabolic disorders [40], and MCP-1 accelerates macrophage infiltration in inflammation accompanying obesity [7]. Up-regulation of gene expression of MCP-1 was found in adipose tissue of obese subjects compared to lean control [41]. Both Es and EsM resulted in significantly down-regulated gene expression of MCP-1 compared to HFD control. This indicates that the endotoxin-induced inflammation was inhibited by alteration of gut microbiota.

In detail, gut microbiota pyrosequencing data showed similar trends at genus level. Blautia, Roseburia, and Clostridium_g6 were selectively enriched by Es and EsM. In particular, most species of Blautia are regarded as short chain fatty acid producers [42]. Hence the enrichment of Blautia is a possible reason for the high level of fecal acetic acid leading to appetite-reduction and eventual weight loss. Blautia is negatively associated with body weight, fat weight, and FBG, different from other genus of gut microbiota (Figure 8). Roseburia was also found to show negative correlation with body weight, consistent with a previous study [43]. In addition, Akkermansia ameliorates HFD induced obesity, metabolic endotoxemia, and glucose homeostasis via change of microbial metabolites, such as SCFA and interference of Foxp3 regulatory T cells $[44,45]$. Our experiment showed that EsM transplantation maintained the restoration of Akkermansia abundance loss. This might be another mechanism to explain why the anti-obesity effect can be maintained only by alteration of gut microbiota transplantation. 
A Family compositions of fecal bacteria

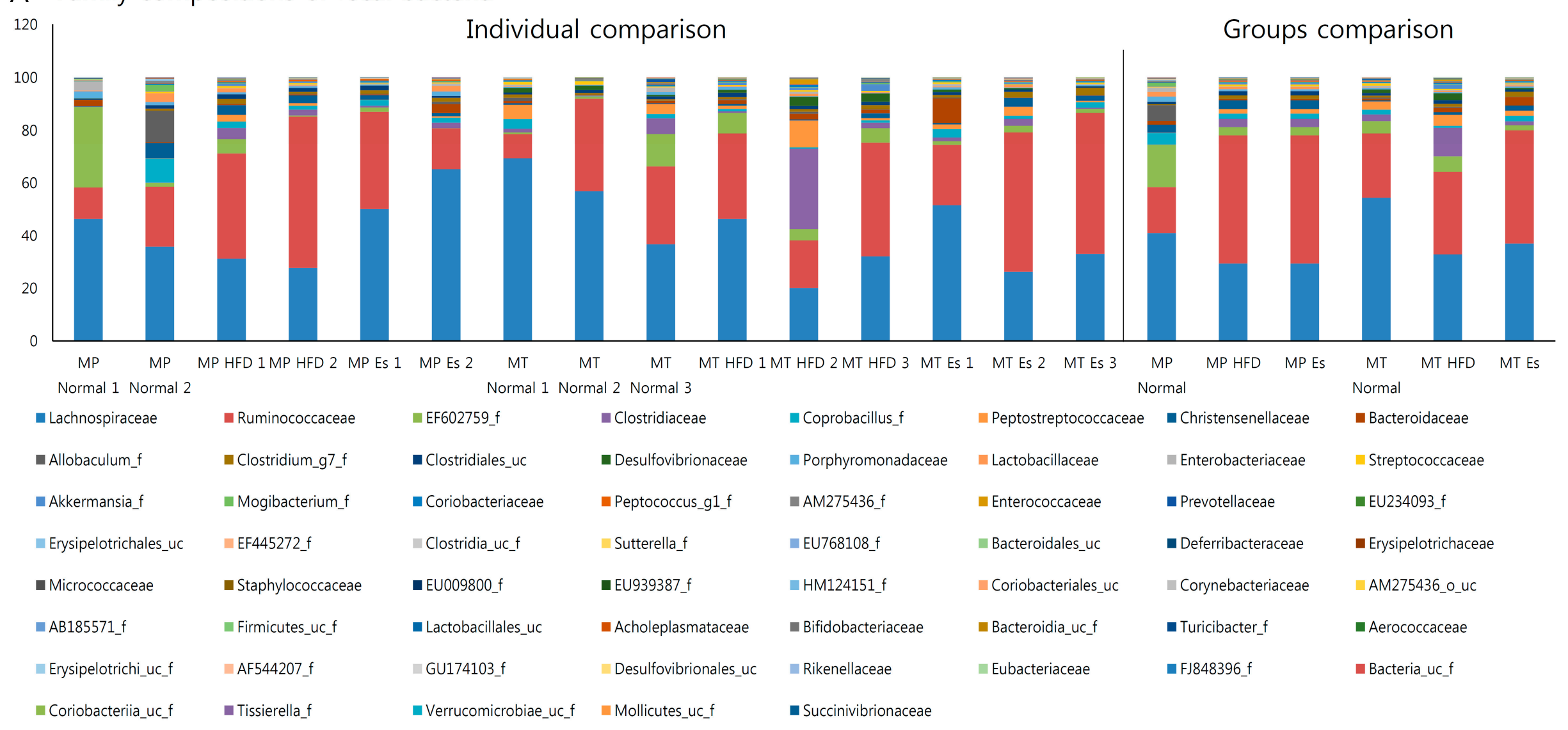

Figure 7. Cont. 
B Genus compositions of fecal bacteria

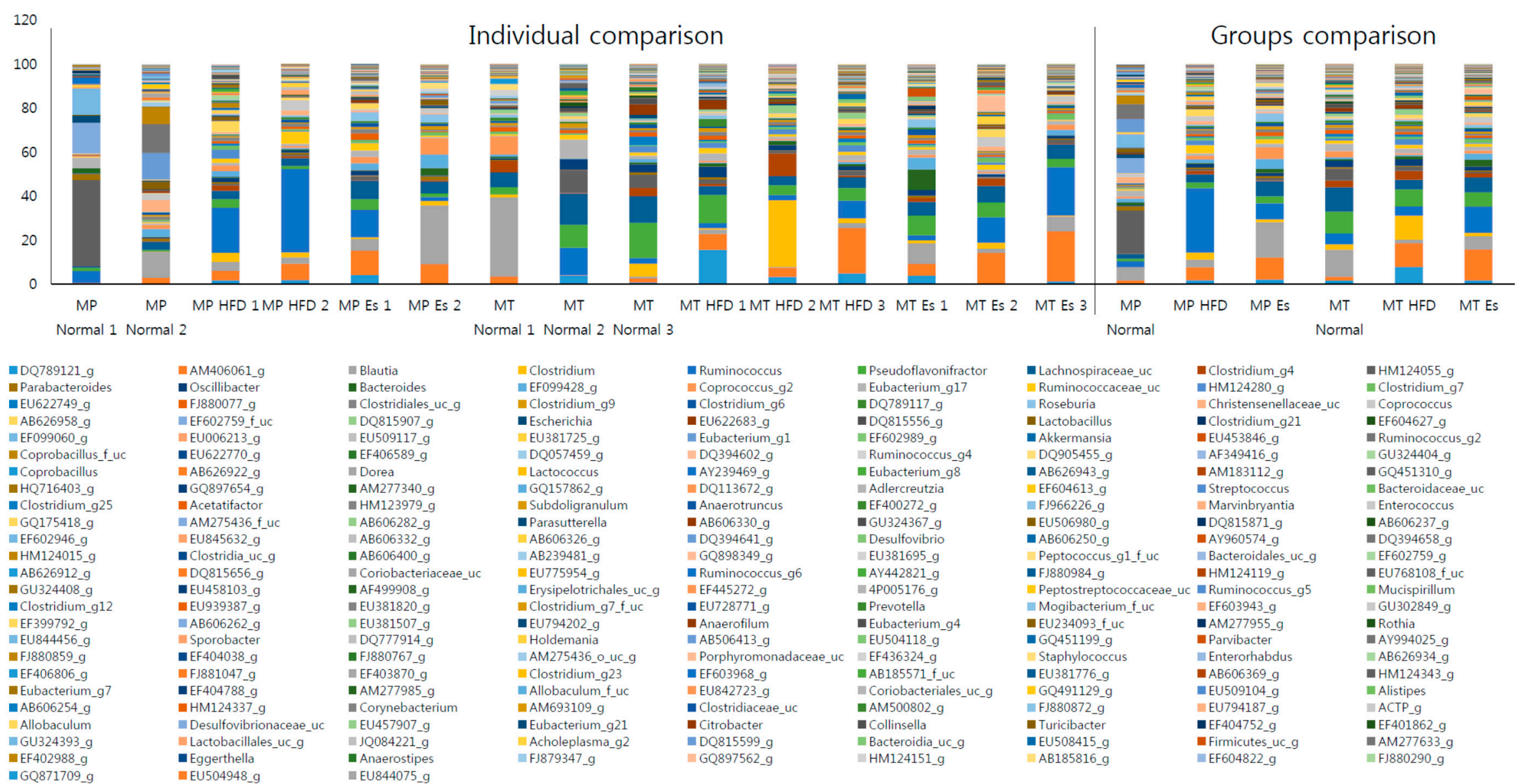

Figure 7. Gut microbiota taxonomic profiles. The relative contributions (\%) of fecal bacterial family (A) and genus (B) were compared both individually and averagely according to the pyrosequencing data. 


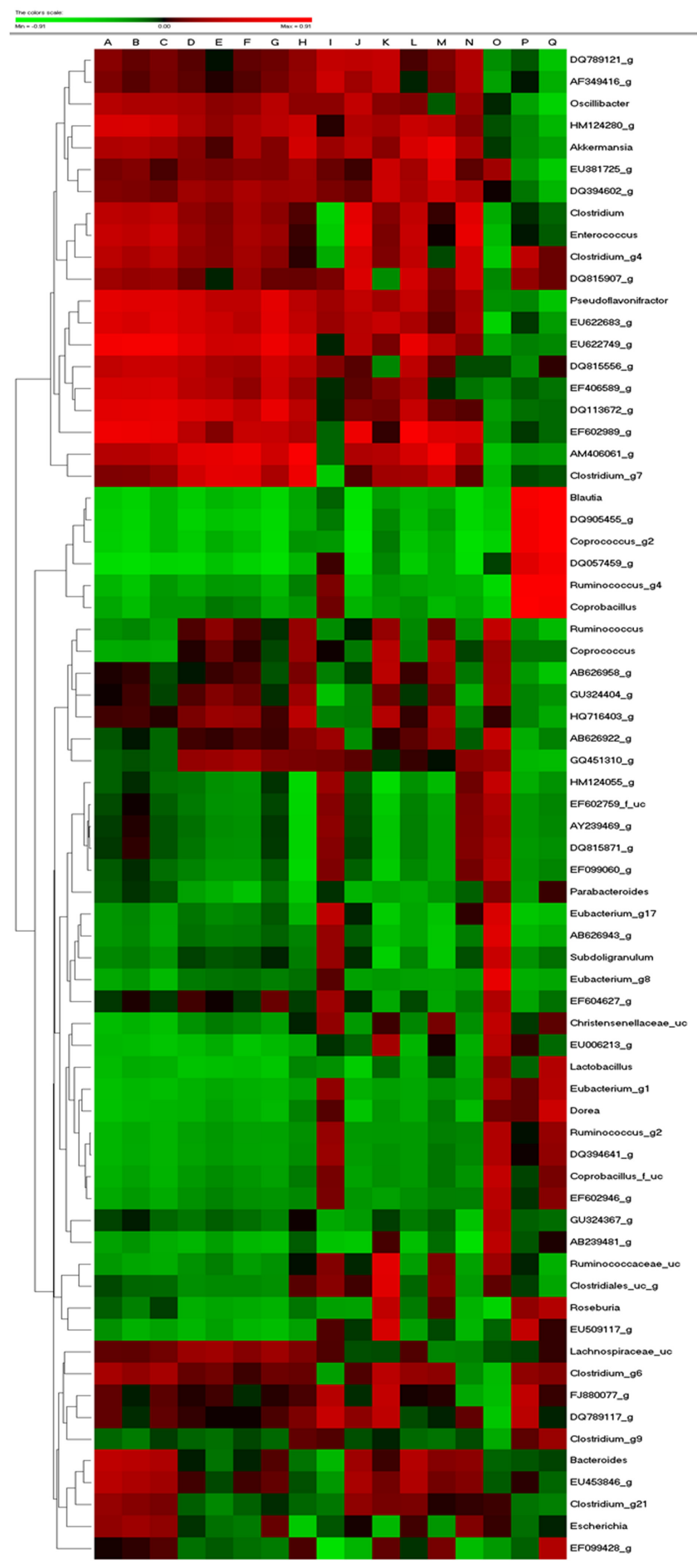

Figure 8. Pearson correlation analysis. All of the data were collected and analyzed using Pearson's correlation score. The scores of Pearson's correlation were figured by PermutMatrix software (Version 1.9.3 EN) using heatmap plots. As the colors scale shown, red color indicates a positive correlation, while green color shows a negative correlation. A: Body weight; B: Body weight gain; C: FER (Food efficiency ratio); D: Total fat; E: AFAT (Abdominal fat); F: EFAT (Epididymal fat); G: PFAT (Perirenal fat); H: Liver; I: AST (aspartate transaminase); J: ALT (alanine transaminase); K: TC (total cholesterol); L: TG (triglyceride); M: FBG (fasting blood-glucose); N: Insulin; O: Food intake; P: HDL (high density lipoprotein); Q: Cecum contents. 
Besides the abovementioned bacteria, body and fat mass were negatively associated with Coprococcus, Ruminococcus, Eubacterium, dorea, and positively associated with Oscillibacter, Chlostridium, Bacteroides, Escherichia. Because of the complex interaction between the variety of gut microbiota and host metabolism [46], multivariate regression analysis is a feasible approach to search for the potential indicators mechanistically connected between the cause and the disease [14]. Therefore, in future it will be worthwhile to confirm if and how these associated bacteria regulate the development of obesity and metabolic disorders via germ free and/or transgenic model. In the MT, normal gut microbiota (NM) was used as a positive control for monitoring transplantation response. Interestingly, both EsM and NM transplant exert the similar amelioration efficacy on HFD induced obesity and metabolic disorders. NM transplant improved most of the metabolic parameters to a greater extent than EsM transplant while EsM transplant was more effective in epididymal fat than NM transplant. As limitation of the study, because the group of normal diet with Es treatment is absent, we are not able to confirm whether normal diet with Es exert synergistic effect via gut microbiota regulation.

\section{Conclusions}

EsM transplantation preserves the amelioration efficacy on HFD induced obesity and metabolic disorders by the association of modulating the distribution of intestinal microbiota, enterogenous endotoxin and enteral acetic acid.

Supplementary Materials: The following are available online at www.mdpi.com/1660-4601/14/6/555/s1, Figure S1: Comparison of gut microbiota, Figure S2: Principal coordinate analysis (PCoA) plots, Figure S3: Rarefaction curves, Table S1: Fecal endotoxin concentration and quality control.

Acknowledgments: This work was supported by the National Research Foundation of Korea Grant funded by the Korean Government (NRF-2016R1A2B4014225).

Author Contributions: H.J.K. designed the study. J.H.W. and K.S.H. performed the experiments. J.H.W. and H.J.K. analyzed the data. JHW and BSK wrote the manuscript. All authors read the final version of manuscript and approved it.

Conflicts of Interest: The authors declare no conflict of interest.

\section{References}

1. Stoner, L.; Cornwall, J. Did the American Medical Association make the correct decision classifying obesity as a disease? Australas. Med. J. 2014, 7, 462-464. [CrossRef] [PubMed]

2. World Health Organization. Obesity: Preventing and Managing the Global Epidemic: Report of a WHO Consultation; World Health Organization, Division of Noncommunicable Disease, Programme of Nutrition Family and Reproductive Health: Geneva, Switzerland, 2000.

3. Hill, J.O.; Wyatt, H.R.; Peters, J.C. Energy balance and obesity. Circulation 2012, 126, 126-132. [CrossRef] [PubMed]

4. Adan, R.A. Mechanisms underlying current and future anti-obesity drugs. Trends Neurosci. 2013, 36, 133-140. [CrossRef] [PubMed]

5. Mohamed-Ali, V.; Pinkney, J.H.; Coppack, S.W. Adipose tissue as an endocrine and paracrine organ. Int. J. Obes. Relat. Metab. Disord. 1998, 22, 1145-1158. [CrossRef] [PubMed]

6. Panee, J. Monocyte Chemoattractant Protein 1 (MCP-1) in obesity and diabetes. Cytokine 2012, 60, 1-12. [CrossRef] [PubMed]

7. Kanda, H.; Tateya, S.; Tamori, Y.; Kotani, K.; Hiasa, K.; Kitazawa, R.; Kitazawa, S.; Miyachi, H.; Maeda, S.; Egashira, K.; et al. MCP-1 contributes to macrophage infiltration into adipose tissue, insulin resistance, and hepatic steatosis in obesity. J. Clin. Investig. 2006, 116, 1494-1505. [CrossRef] [PubMed]

8. Visser, M.; Bouter, L.M.; McQuillan, G.M.; Wener, M.H.; Harris, T.B. Elevated C-reactive protein levels in overweight and obese adults. JAMA 1999, 282, 2131-2135. [CrossRef] [PubMed]

9. Fain, J.N.; Madan, A.K.; Hiler, M.L.; Cheema, P.; Bahouth, S.W. Comparison of the release of adipokines by adipose tissue, adipose tissue matrix, and adipocytes from visceral and subcutaneous abdominal adipose tissues of obese humans. Endocrinology 2004, 145, 2273-2282. [CrossRef] [PubMed] 
10. DeBoer, M.D. Obesity, systemic inflammation, and increased risk for cardiovascular disease and diabetes among adolescents: A need for screening tools to target interventions. Nutrition 2013, 29, 379-386. [CrossRef] [PubMed]

11. Kim, K.A.; Gu, W.; Lee, I.A.; Joh, E.H.; Kim, D.H. High fat diet-induced gut microbiota exacerbates inflammation and obesity in mice via the TLR4 signaling pathway. PLoS ONE 2012, 7, e47713. [CrossRef] [PubMed]

12. Cani, P.D.; Amar, J.; Iglesias, M.A.; Poggi, M.; Knauf, C.; Bastelica, D.; Neyrinck, A.M.; Fava, F.; Tuohy, K.M.; Chabo, C.; et al. Metabolic endotoxemia initiates obesity and insulin resistance. Diabetes 2007, 56, 1761-1772. [CrossRef] [PubMed]

13. Cani, P.D.; Bibiloni, R.; Knauf, C.; Waget, A.; Neyrinck, A.M.; Delzenne, N.M.; Burcelin, R. Changes in gut microbiota control metabolic endotoxemia-induced inflammation in high-fat diet-induced obesity and diabetes in mice. Diabetes 2008, 57, 1470-1481. [CrossRef] [PubMed]

14. Zhao, L. The gut microbiota and obesity: From correlation to causality. Nat. Rev. Microbiol. 2013, 11, 639-647. [CrossRef] [PubMed]

15. Alang, N.; Kelly, C.R. Weight gain after fecal microbiota transplantation. Open Forum Infect. Dis. 2015, 2, ofv004. [CrossRef] [PubMed]

16. Kumar, H.; Lund, R.; Laiho, A.; Lundelin, K.; Ley, R.E.; Isolauri, E.; Salminen, S. Gut microbiota as an epigenetic regulator: Pilot study based on whole-genome methylation analysis. mBio 2014, 5, e02113-14. [CrossRef] [PubMed]

17. Wang, J.H.; Bose, S.; Kim, G.C.; Hong, S.U.; Kim, J.H.; Kim, J.E.; Kim, H. Flos Lonicera ameliorates obesity and associated endotoxemia in rats through modulation of gut permeability and intestinal microbiota. PLoS ONE 2014, 9, e86117. [CrossRef] [PubMed]

18. Xie, W.; Gu, D.; Li, J.; Cui, K.; Zhang, Y. Effects and action mechanisms of berberine and Rhizoma coptidis on gut microbes and obesity in high-fat diet-fed C57BL/6J mice. PLoS ONE 2011, 6, e24520. [CrossRef] [PubMed]

19. Xu, J.; Lian, F.; Zhao, L.; Zhao, Y.; Chen, X.; Zhang, X.; Guo, Y.; Zhang, C.; Zhou, Q.; Xue, Z.; et al. Structural modulation of gut microbiota during alleviation of type 2 diabetes with a Chinese herbal formula. ISME J. 2014, 9, 552-562. [CrossRef] [PubMed]

20. Zhang, X.; Zhao, Y.; Zhang, M.; Pang, X.; Xu, J.; Kang, C.; Li, M.; Zhang, C.; Zhang, Z.; Zhang, Y.; et al. Structural changes of gut microbiota during berberine-mediated prevention of obesity and insulin resistance in high-fat diet-fed rats. PLoS ONE 2012, 7, e42529. [CrossRef] [PubMed]

21. Chen, K.K. A comparative study of synthetic and natural ephedrines. J. Pharmacol. Exp. Ther. 1928, 33, $237-258$.

22. Shekelle, P.G.; Hardy, M.L.; Morton, S.C.; Maglione, M.; Mojica, W.A.; Suttorp, M.J.; Rhodes, S.L.; Jungvig, L.; Gagne, J. Efficacy and safety of ephedra and ephedrine for weight loss and athletic performance: A meta-analysis. JAMA 2003, 289, 1537-1545. [CrossRef] [PubMed]

23. Song, M.K.; Um, J.Y.; Jang, H.J.; Lee, B.C. Beneficial effect of dietary Ephedra sinica on obesity and glucose intolerance in high-fat diet-fed mice. Exp. Ther. Med. 2012, 3, 707-712. [PubMed]

24. Kim, B.S.; Song, M.Y.; Kim, H. The anti-obesity effect of Ephedra sinica through modulation of gut microbiota in obese Korean women. J. Ethnopharmacol. 2014, 152, 532-539. [CrossRef] [PubMed]

25. Matthews, D.R.; Hosker, J.P.; Rudenski, A.S.; Naylor, B.A.; Treacher, D.F.; Turner, R.C. Homeostasis model assessment: Insulin resistance and beta-cell function from fasting plasma glucose and insulin concentrations in man. Diabetologia 1985, 28, 412-419. [CrossRef] [PubMed]

26. United States Pharmacopeial Convention. General Chapter <85> Bacterial Endotoxins Test; United States Pharmacopeia (USP): Rockville, MD, USA, 2012.

27. Langille, M.G.; Zaneveld, J.; Caporaso, J.G.; McDonald, D.; Knights, D.; Reyes, J.A.; Clemente, J.C.; Burkepile, D.E.; Vega Thurber, R.L.; Knight, R.; et al. Predictive functional profiling of microbial communities using 16S rRNA marker gene sequences. Nat. Biotechnol. 2013, 31, 814-821. [CrossRef] [PubMed]

28. Abubucker, S.; Segata, N.; Goll, J.; Schubert, A.M.; Izard, J.; Cantarel, B.L.; Rodriguez-Mueller, B.; Zucker, J.; Thiagarajan, M.; Henrissat, B.; et al. Metabolic reconstruction for metagenomic data and its application to the human microbiome. PLoS Comput. Biol. 2012, 8, e1002358. [CrossRef] [PubMed]

29. Ng, M.; Fleming, T.; Robinson, M.; Thomson, B.; Graetz, N.; Margono, C.; Mullany, E.C.; Biryukov, S.; Abbafati, C.; Abera, S.F.; et al. Global, regional, and national prevalence of overweight and obesity in children and adults during 1980-2013: A systematic analysis for the Global Burden of Disease Study 2013. Lancet 2014, 384, 766-781. [CrossRef] 
30. Daniel, H.; Moghaddas Gholami, A.; Berry, D.; Desmarchelier, C.; Hahne, H.; Loh, G.; Mondot, S.; Lepage, P.; Rothballer, M.; Walker, A.; et al. High-fat diet alters gut microbiota physiology in mice. ISME J. 2014, 8 , 295-308. [CrossRef] [PubMed]

31. Woting, A.; Pfeiffer, N.; Loh, G.; Klaus, S.; Blaut, M. Clostridium ramosum promotes high-fat diet-induced obesity in gnotobiotic mouse models. MBio 2014, 5, e01530-14. [CrossRef] [PubMed]

32. Bäckhed, F.; Ding, H.; Wang, T.; Hooper, L.V.; Koh, G.Y.; Nagy, A.; Semenkovich, C.F.; Gordon, J.I. The gut microbiota as an environmental factor that regulates fat storage. Proc. Natl. Acad. Sci. USA 2004, 101, 15718-15723. [CrossRef] [PubMed]

33. Wang, J.H.; Bose, S.; Kim, H.G.; Han, K.S.; Kim, H. Fermented Rhizoma Atractylodis Macrocephalae alleviates high fat diet-induced obesity in association with regulation of intestinal permeability and microbiota in rats. Sci. Rep. 2015, 5, 8391. [CrossRef] [PubMed]

34. Turnbaugh, P.J.; Backhed, F.; Fulton, L.; Gordon, J.I. Diet-induced obesity is linked to marked but reversible alterations in the mouse distal gut microbiome. Cell Host Microbe 2008, 3, 213-223. [CrossRef] [PubMed]

35. Frost, G.; Sleeth, M.L.; Sahuri-Arisoylu, M.; Lizarbe, B.; Cerdan, S.; Brody, L.; Anastasovska, J.; Ghourab, S.; Hankir, M.; Zhang, S.; et al. The short-chain fatty acid acetate reduces appetite via a central homeostatic mechanism. Nat. Commun. 2014, 5, 3611. [CrossRef] [PubMed]

36. Wang, J.; Tang, H.; Zhang, C.; Zhao, Y.; Derrien, M.; Rocher, E.; Van-Hylckama Vlieg, J.E.; Strissel, K.; Zhao, L.; Obin, M.; et al. Modulation of gut microbiota during probiotic-mediated attenuation of metabolic syndrome in high fat diet-fed mice. ISME J. 2015, 9, 1-15. [CrossRef] [PubMed]

37. Yamashita, H.; Fujisawa, K.; Ito, E.; Idei, S.; Kawaguchi, N.; Kimoto, M.; Hiemori, M.; Tsuji, H. Improvement of obesity and glucose tolerance by acetate in Type 2 diabetic Otsuka Long-Evans Tokushima Fatty (OLETF) rats. Biosci. Biotechnol. Biochem. 2007, 71, 1236-1243. [CrossRef] [PubMed]

38. Everard, A.; Lazarevic, V.; Derrien, M.; Girard, M.; Muccioli, G.G.; Neyrinck, A.M.; Possemiers, S.; Van Holle, A.; Francois, P.; De Vos, W.M.; et al. Responses of gut microbiota and glucose and lipid metabolism to prebiotics in genetic obese and diet-induced leptin-resistant mice. Diabetes 2011, 60, 2775-2786. [CrossRef] [PubMed]

39. Hwang, I.; Park, Y.J.; Kim, Y.R.; Kim, Y.N.; Ka, S.; Lee, H.Y.; Seong, J.K.; Seok, Y.J.; Kim, J.B. Alteration of gut microbiota by vancomycin and bacitracin improves insulin resistance via glucagon-like peptide 1 in diet-induced obesity. FASEB J. 2015, 29, 2397-2411. [CrossRef] [PubMed]

40. Nov, O.; Shapiro, H.; Ovadia, H.; Tarnovscki, T.; Dvir, I.; Shemesh, E.; Kovsan, J.; Shelef, I.; Carmi, Y.; Voronov, E.; et al. Interleukin-1 $\beta$ regulates fat-liver crosstalk in obesity by auto-paracrine modulation of adipose tissue inflammation and expandability. PLoS ONE 2013, 8, e53626. [CrossRef] [PubMed]

41. Huber, J.; Kiefer, F.W.; Zeyda, M.; Ludvik, B.; Silberhumer, G.R.; Prager, G.; Zlabinger, G.J.; Stulnig, T.M. CC chemokine and $\mathrm{CC}$ chemokine receptor profiles in visceral and subcutaneous adipose tissue are altered in human obesity. J. Clin. Endocrinol. Metab. 2008, 93, 3215-3221. [CrossRef] [PubMed]

42. Park, S.K.; Kim, M.S.; Roh, S.W.; Bae, J.W. Blautia stercoris sp. nov., isolated from human faeces. Int. J. Syst. Evol. Microbiol. 2012, 62, 776-779. [CrossRef] [PubMed]

43. Conterno, L.; Fava, F.; Viola, R.; Tuohy, K.M. Obesity and the gut microbiota: Does up-regulating colonic fermentation protect against obesity and metabolic disease? Genes Nutr. 2011, 6, 241-260. [CrossRef] [PubMed]

44. Everard, A.; Belzer, C.; Geurts, L.; Ouwerkerk, J.P.; Druart, C.; Bindels, L.B.; Guiot, Y.; Derrien, M.; Muccioli, G.G.; Delzenne, N.M.; et al. Cross-talk between Akkermansia muciniphila and intestinal epithelium controls diet-induced obesity. Proc. Natl. Acad. Sci. USA 2013, 110, 9066-9071. [CrossRef] [PubMed]

45. Smith, P.M.; Howitt, M.R.; Panikov, N.; Michaud, M.; Gallini, C.A.; Bohlooly, Y.M.; Glickman, J.N.; Garrett, W.S. The microbial metabolites, short-chain fatty acids, regulate colonic Treg cell homeostasis. Science 2013, 341, 569-573. [CrossRef] [PubMed]

46. Tremaroli, V.; Backhed, F. Functional interactions between the gut microbiota and host metabolism. Nature 2012, 489, 242-249. [CrossRef] [PubMed]

(c) 2017 by the authors. Licensee MDPI, Basel, Switzerland. This article is an open access article distributed under the terms and conditions of the Creative Commons Attribution (CC BY) license (http:/ / creativecommons.org/licenses/by/4.0/). 